\title{
Nanotechnology Combating Fight against Cancer
}

\author{
Khalid R*, Sabir L, Qurat ul ain Haider, Aslam S and Ameer S \\ Institute of Molecular Biology \& Biotechnology Bahauddin Zakariya University, \\ Pakistan
}

\section{Review Article \\ Volume 2 Issue 1}

Received Date: May 08, 2019

Published Date: June 26, 2019

DOI: $10.23880 /$ aabsc- 16000130

*Corresponding author: Rana Khalid Iqbal, Institute of Molecular Biology \& Biotechnology Bahauddin Zakariya University, Multan-60800, Pakistan, Email: khalid.iqbal@bzu.edu.pk

\begin{abstract}
Cancer is a major cause of death worldwide. Cancer spread through multiple steps that make it an incurable disease. Earlier different conventional methods are used such as radiotherapy, chemotherapy, and immunotherapy to treat cancer. In conventional also affects the normal body cells. To overcome this problem different new approaches are applied that help to diagnose and treat cancer. With the advancement of techniques, new technique emerges called cancer nanotechnology that helps to diagnose and treat cancer by using different drug delivery system. Cancer nanotechnology uses different nanoparticles or nano vectors as a carrier to treat the disease. These nano-carriers for drug delivery include liposomes, polymers, dendrimers, micelles, carbon nano-tube, quantum dots, etc. Active and passive targeting helps to target the drug and easily recognize the site of the tumor. The aim of this paper is to provide information about how cancer nanotechnology helps in combating the fight against cancer. And also the use of different drug delivery systems to treat and recognize cancer.
\end{abstract}

Keywords: Nanotechnology; Nanoparticles; Nano Drugs; Nanocarrier; Tumor Imaging

\section{Introduction}

Cancer is the major cause of death worldwide. Cancer nanotechnology allows treating and prevents from cancer. Cancer starts as a localized disease but spread through multiple steps that make its incurable disease. Cancer treatment has been performed on the basis of clinical and pathological staging, most common cancer treatment is restricted to chemotherapy, radiation, and surgery [1]. Cancer is inherently a nano-structural problem. There seems obvious benefits to treatment apply at nanoscale structures. Nanotechnology is a new fields of biotechnology those open-ups the new era of prevention from harmful diseases such as cancer. Nanotechnology develops rapidly which aims to provide a forum so that most promising emerging themes which were pre- eminent in the minds of researchers. Nanotechnology includes nano-scale processes and technology that helps in the intersection of cancer research, encompassing prevention, treatment diagnosis, and related regulatory framework [2]. Nanotechnology involves object with characteristics length scales typically below $500 \mathrm{~nm}$ [2]. Cancerous cell are vulnerable which causes side effects like hair loss, vomiting, nausea, etc.

Nanotechnology creates very small nanoparticles device system of $1 \mathrm{~nm}$ equals 1 billionth of a meter, the width is about 5 atoms. They are designed in such a way so that they may interact with cell tissue at a molecular level with high specificity. The word Nano is derived from the Greek word 'Nanos' which means dwarf. Nanotechnology aims at manipulating atoms molecules 
and nanosize particles in a precise and controlled manner to build with nano-properties [3]. They are simply the assembly of atoms. Nanotechnology is also called 'bottomup technology' build by the block of different atoms [4]. Advancement in nano-materials and design of nanocarrier helps in several applications like therapy, diagnosis and therapeutic [2]. Breast cancer has a higher number of nano-medicine. More than 50 companies have been working for the nano-medicine for the treatment of breast cancer [5]. By using cancer nanotechnology techniques we can detect early sign of tumor or malignancy by simple routine screening by proteome pattern analysis from a blood sample and examine the molecular profiling of patients in vivo imaging. Nanotechnology detects the transformed cell populations early by in-vivo imaging or ex-vivo analysis. It is allowed to detect early cancer and to allow early cancer to eliminate effecting healthy tissue and monitoring the treatment effects in real time [6]. Cancer nanotechnology improves the approaches to detect diagnose imaging and therapy and it also reduces the toxicity associated with traditional cancer therapy. Cancer nanotechnology application of nanotechnology towards monitoring repair and improvement of the human biological system related to cancer. For routine clinical trials, nanotechnology requires multi-disciplinary approaches to releases social or ethical issues. Nanotechnology includes devices such as nano-vector or nanocantilever array which are among the leading approach under development for detection of precancerous and malignant lesions from biological fluids cancer-related examples of it's include injectable drugs delivery nano-vector or such as liposomes for therapy of cancer [7]. Nanotechnology concerns with the study of the component in the $1-1000 \mathrm{~nm}$ dimensional range. Drug delivery and imaging nano-vector intravascularly, injectable nano vector may be used for detection or diagnosis of cancer [6]. Nanomedicine as multimodulatory treatment of cancer utilizes the advantage of the nano-delivery system. Multi-modularity requires a different therapeutic agent as an exact ratio. Balance ratio in the formulation of nano-medicine along with nanocarriers for effective oncology treatment. For example combine effect of docetaxel and cisplatin show enhanced the effect on tumor destruction than in separate usage [8].

\section{Properties of Nanoparticles}

Nanoparticles possess unique properties like mechanical strength, electrical conductivity, thermal conductivity, chemical reactivity, and transparency [9]. They can be of Nanoscale thin film/ ultra-thin film, Nanotubes/ nanowires/ nanorods, Nano structural material. Nanoscale devices are 100-100,000 times smallest than human cells. The diameter of human hair is about $80,000 \mathrm{~nm}$ while that of DNA molecule is between 2 $\mathrm{nm}$ to $12 \mathrm{~nm}$. Nanodevices are of less than $100 \mathrm{~nm}$ can enter into cells and organelles inside and interact with DNA and proteins. Nanotools are entered into a cell and monitor the cell. They help in detection of earliest stage cancer. Nanoparticles drugs are designed by encapsulating, covalently attach or adsorbing of a therapeutic and diagnostic agent to nanoparticles $[4,10]$. Nanovector is constituted of three major components such as featuring or core constituent's material, a therapeutic and imaging payload, and biological surface modifier with help in targeting tumor [11]. The advantage of using nano-vector enhances the specificity of drug delivery that we can target disease abnormal part and replace with normal that depend on the size and physical properties of Nano-vector. It is estimated that several thousands of different nano vector have been reported in the literature but the only few of them are using fighting against cancer.

The advantage of employing drug delivery to cancer:

- Nano-carrier permit delivery of poorly soluble drugs

- Nanocarrier can be functionalized with various molecules such as one or more drug, an imaging agent and PEG through adsorption and chemical conjugation

- Nanocarrier within the range of 20-400nm can target cancer site through passive targeting, enhanced permeability

- Nanocarrier does not carry the one drug but it can also be loaded with the multiple drugs, but if they are designed to fight cancer through functionalization with target moieties, so this nano-carrier serves as "magic bullets". Targeting moieties promote targeting of cancer cell by knowing about the molecule expressed by a cancer cell. For example, folate receptor alpha(Fra) expressed by epithelial cancer which is a tumor marker, but in normal it is not accessible through blood

- Nanocarrier modification with PEG prolong their blood circulation time

- Nanocarrier promotes overcoming drug resistance through more than one mechanism

- Nano-carrier increases the safety and tolerability of drugs [6].

- Nanoparticles itself has therapeutic or diagnostic properties can be designed to carry a target therapeutic payload.

- Nanoparticles attached to high affinity and specificity multivalent targeting ligands for a target cell.

- Nanoparticles accommodate multiple drugs and allow treating cancer.

- Nanoparticles bypass the traditional drug resistance mechanism [12]. 
- Nanoparticles have size like viruses. They are nonimmunogenic, easily taken up by macrophages and antigen presenting cells which dominate the traditional cancer immunotherapy. Thus improves the survival rate [13].

- Nanoparticles such as liposomesmicellus and polymeric complex possess pharmacokinetic properties. The disruption in morphology allows uptake of nanoparticle e.g. abnormal vasculature possess aberrant branching component and leaky arterial walls cause by the proliferation of malignant cells. Thus allow easily infiltration of nanoparticles [14]. After specifying engineered nanomedicine possess unique properties and are capable of biodistribution of a drug through passive targeting efficiently e.g the nano-encapsulation of loaded nanomedicine can prevent by cell internal environment and bind to the specific target and release precise dose to the tumor [15].

- Nanoparticle enhances permeability and EPR. A specific range of nanoparticles 100-1000 $\mathrm{nm}$ accumulates in tissues. Tumor physiology helps in secretion of enhancing vesicular endothelial growth receptors and induces angiogenesis. Vascularization in cancer produce vessels with large ends gap leads to easier transport of macromolecules through vessels. Lymphatic vessels are less developed in tumor contribute accumulations. Lymphatic nodes cause nonspecific accumulation [16].

\section{Immunotherapy}

Chemotherapy is the conventional method for treatment of cancer but this method is nonspecific and leads to toxicity but the new technology to improves the problems hurdles such as it reduce toxicity improves pharmacokinetics and bioavailability these are advantages over conventional cancer treatment [17]. Immunotherapy is the most effective method of treatment in cancer which enhances the patient immune system against cancer and reduces side effects. It used along with various nano-drugs. In past therapies like Monoclonal antibody target tumor antigen alone with chemotherapy and radiotherapy used show significant effect on oncology. It helps in the immune system so that in combat fight against cancer. In old therapeutic methods, only small dose reach to target site so that a higher amount of dose given to patients which may lead to immunogenic reactions due to the interaction of the immune therapeutic agent with the immune system. Nowadays, nanotechnology has proved successfully develop better drug delivery system [16]. Cancer immunotherapy tried to overcome the hurdle such as multititer immune suppressive mechanisms with the collaboration of nano- vaccine [18]. Nanotechnology enhances the immune survivals which activate co-stimulatory pathways to counteract the tumor-mediated inhibitor.

Cancer immune therapies can be: A Cell-free therapy which direct administration of the therapeutic agent to tumor-specific antigen and kills the cancer cells, Cellbased therapies collect and grows cancer targets immune cell from the patient and causes the destruction of the tumor. eg: chimeric antigen receptor $\mathrm{T}$ [16]. The size, surface chemistry, and architecture of nano-particles confer distinct bio-properties and enable transport of diverse payload with high efficiency. Nanoscale platform explores nano opportunity in cancer immunotherapy, tools for integration of cancer in vivo and overcoming traditional barriers to cancer therapy. Cancer immunotherapy, checkpoint inhibitor therapies, cellbased CAR T-cell therapies have improved survival in the treatment of melanoma, bladder, renal and nano small cell lung cancer. Inherent multi-component cargo capacity of nano-scale delivery platforms enable an alternative approach in cancer immunotherapy to boost up response [13].

\section{Radiotherapy}

Radiotherapy is an area of science provides a new avenue to impact radiation oncology in nanomedicine. Nanoparticles have high retention effect with large permeability and magnetism on the tumor cells. Though radiation may cause abnormalities in the DNA there are several radio techniques have been developed for anticancer treatment, still, challenges remain. eg: chemoradiotherapy may cause a toxic effect in the body but help in reduction of mortality. Nanosize therapeutic agent circulates in the blood system and thus diffuse against the target cell as the physiology of normal tissue disturbs in tumor cells, they infiltrate to target cells and accumulate in tumor cells [14].

Diagnosis of cancer can be done by bio-sensing or diagnostic imaging. Bio-sensing detects bio-molecules that act as a biomarker that help in detecting cancer and circulating cancer cell. Imaging detects cancer cell that helps in the diagnosis of cancer and also helps during surgery to detect the cancer tissue and also size and type of cancer [6]. Radioisotope imaging is an emerging technology over CT and MRI provide better diagnosis value in oncology eg: fluorodeoxyglucose is radioactive using in research. Radio-sensitizing NPs have effective tumor control in oncology therapy combines X-ray with a high atomic number shows strong photoelectric absorption of x-ray which increases distribution of malignant cell [19]. Radiosensitive nano-particles 
extensively used in cancer research. Modulated Radiation Therapy and volumetric modulated ARC Therapy, IGRI radiotherapy with a combined effect of NPs are in development adopting new avenues of research [19]. Versatility of nano-particles provide potential to probe critical cancer targets and identify imaging biomarkers in clinical trials, eg: targeted particle radiotherapies and use of radio-sensitizing nano-material with high atomic number $\mathrm{Z}$ enhance the efficiency of radiation to tumor [13]. The MRI technique along with Fluorescence imaging and confocal scanning microscopy show excellent tools for many in vitro studies on a tumor. Nanocomplex formed by conjugation along with antibodies targets the human epidermal growth factor expressed in breast cancer provide the information related to the distribution of nanomaterial. Other imaging nanomaterials such as polyacid nanoparticles, mesoporous silica nanoparticles, liposomes silica AuNSs, gold nanorods, carbon tubes; nano globules, graphene oxide, and nanodiamond have been studied for breast tissue imaging and cancer therapy [5].

In Radioisotope delivery radionuclide emit energy and have the ability to cleave the DNA. B-emitters have penetration power to tissues and have lower energy used in oncology treatment, eg: Re 186, Re188, Sr89, p32, and y90 are nano nuclei used. Radioisotopes along with nanocarrier are used for the effective treatment. Radios nanoparticles are most suitable for the treatment and avoid biodistribution.cu64 labeled isotopes suppress the malignancy in a breast. $50 \mathrm{~nm}$ lipid nanocapsule along with Re188 used in internal radiotherapy of glioblastoma.

Radiosensitizer along with nanoparticles formulation improves the delivery of nanomedicine which has low distribution to normal cells, eg: Histone deacetylase inhibitor is effective in colorectal and prostate cancer. Gadolin and gold-based nanoparticles used as radiosensitizers effectively break DNA and reduced tumor growth. The image guided radiotherapy eg: computed tomography (CT), MRI, ultrasound, x-ray imaging precisely used in oncology treatment. Nanoparticles enhance the guided radiotherapy techniques. Nanoparticles such as liposomesmicellus and polymeric complex possess pharmacokinetic properties. The disruption in morphology allows uptake of nanoparticle eg: abnormal vasculature possess aberrant branching component and leaky arterial walls cause by the proliferation of malignant cells. Thus allow easily infiltration of nanoparticles [14].

Clinical cancer imaging technologies do not provide complete information about cancer profiling to identify malignancy based on their molecular expression profiling used. Nanoparticle probes are used for molecular profiling and provide information about the spread and distribution of cancer and marker associated with tumor microenvironment. Angiogenesis is an important marker found as premalignant lesions of cervix, breast, and skin and detect cancer [20]. In Ct image nanoparticles have been formulated in such a way so that they can exploit high $\mathrm{Z}$ elements, such as Gold and Platinum. They show high absorption and biocompatibility. They rapidly circulate in blood because they have the ability to penetrate leaky vasculature when delivered intravenously. Tumor-specific contrast CT imaging can be achieved by combing NPs with a suitable antibody or peptide.

Radioisotope imaging is an emerging technology over CT and MRI provide better diagnostic value in oncology, eg: Fluorodeoxyglucose radioactive is using in research. In Radiation therapy, radio sensitizing NPs have effective tumor control in oncology treatment. Combining x-ray along with NPs with high $\mathrm{Z}$ show strong photoelectric absorption of $\mathrm{x}$-ray to tumor cells causing enhance destruction of cancer cells. Modulated Radiation Therapy, VolumatricModualted Arc Theprayand IGRT radiotherapy with the combined effect of NPs is in development, opening new avenues for research. Approximately 70 radiotherapy centers have been developed [19]. Several types of nanoparticles has been used in medical sciences for magnetic resonance imaging(MRI) that include iron oxide-based nanoparticles multiple mode imaging contrast newsagents that combine magnetic resonances with biological targeting and optical detection. For the enhancement of ultrasound imaging low-density lipid nanoparticles has been used [20].

\section{Tools of Nanotechnology}

More complex nanocarrier include micelles (made up of a self-assembling molecule having hydrophobic and hydrophilic segment) hydrogel and dendrimers (made up of the natural and non-natural component). Some new nanocarrier introduce include viruses, metal or oxide based nanocarrier (gold, magnetic or quantum, silica). Carbon-based nanocarrier includes nanotube and fullerenes, help in biomedical application [21]. These nanocarriers deliver tumor-specific antigens to an immune cell that elicit an immune response against cancer [6].

\section{Liposomes}

The liposome is the first generation nanocarrier made up of phospholipids having a polar head and hydrophobic 
tail. Drug delivery can also be functionalized by PEGylation with a molecular weight between 1 to $40 \mathrm{KDa}$ to deliver hydrophobic or hydrophilic drugs $[6,22]$. Liposomes are a versatile tool in biology example of liposome mediate drug deliveries are doxorubicin (Doxil) and daunorubicin (dounoxome) that currently marketed as a liposome delivery system [23]. PEGylated liposome doxorubicin has achieved the most prolonged circulation time with a half-life of 55 hours in human [1]. Liposomes nanoparticles are formed by the encapsulation to increase the pharmacokinetics, eg: Mycet, calyx, Thermodox are currently or trial basis for breast cancer treatment like doxorubicin (dioxin) opened a new way to nanomedicine [24]. These are under development. Liposome with cationic lipid serves as an adjuvant. A little modification of nanoparticles enhances bioavailability and therapeutic efficiency [16].

They have the affinity with the cell membrane, easily infiltrate to tumor cells, high compatibility. Liposomes have still deficiency due to large size, low stability, and low loading efficiency. This is improved by the development of polymeric liposomes which significantly improves the drug delivery system [8]. Archetypal form of nano, the vector is liposome that they use to detect the cancer site and increase drugs concentration at the tumor site. Before treatment of Kaposi sarcoma liposome encapsulation formulation of doxorubicin used for the treatment of cancer. The liposome is the first nanovector for more efficient drugs delivery metabolites [20].

\section{Polymers}

The simple polymer also includes in the first generation of drug nanocarrier, they used to deliver nonspecific drugs after chemically conjugated them to the polymer such as natural polymer polysaccharides, amino acids sugars, and polypeptides. Examples of such polysaccharides include chitosan and albumin, in addition to polylactic acid and poly-glycolic acid or combination of both. Liposome and polymer prove advantageous because of biocompatibility and biodegradability [6].

\section{Polymeric Nanoparticle}

Polymeric nanoparticle submicron size colloidal particle in which anticancer agent encapsulated or conjugated other or onto the surface of PNPS polymeric coated nanoparticle help to remove the cancer cells by working with site-specific targets this method having many advantages over other cancer treatment method. That includes avoiding the screening of speed sharing. Sulfactant and chlorinated solvents (a) Emission of organic solvent case (b) High drug encapsulation efficiency (c) Seal up procedure. Polymer shell provides (polyvinyl alcohol PVA) polyethylene glycol PEG, polysulfate provide protection against the degradation activities of body enzyme-polymer provides the protection of hydrophilic chain over nanoparticles from diffusion hydrolysis and enzymatic degradation that cause the release of an anticancer agent from PNPS [25]. Additionally, anticancer agent coated with PNPS based targeted drug delivery system (TDDS) increase the tissuespecific dry delivery through active and passive tumor targeting without inflating healthy cells [26]. They are prepared with biodegradable and formed by polymer along with the nanocarriers in the aqueous solvent, eg: polylactic acid, polylactic-co-glycolic acid, and polycaprolactone. The technique modifies for effective as the hydrophilicity of the polymer becomes a hindrance. Polymeric nanoparticle along with nanocarriers loaded through chemical conjugation or physical adsorption. The size may vary from 10 to $100 \mathrm{~nm}$. Hydrophilicity avoid by loading hydrophobic therapeutic agent through conjugating electro statically adsorption to the hydrophobic therapeutic agent [8].

\section{Dendrimers}

Dendrimers are the large synthetically dendrite-like polymer, classified depends on the number of branches. They are approximately 2-10 $\mathrm{nm}$. They show reproductive pharmacokinetics have structure and molecular weight [27]. They have higher EPR and are nonimunogenic, eg: dendrimer conjugated magnetic nanoparticles have been designed against breast cancer [24]. They are man-made molecule about the size of an average protein. The surface area provides an attachment of the therapeutic agent and bioactive compound [4]. They are symmetrical and spherical macromolecules have the dendrite-like structure they have high uniformity, rapid cellular entry, reduce macrophage uptake, high target availability, high loading efficiency of nano-delivery [8]. Dendrimers are a self-assembling synthetic polymer and used to detect cancer cell in a lymph node in patients and provide information about the metastatic spread and early signal of disease [20].

\section{Micelles}

Micelles nano complex are novel drug delivery system which enhances the anti-cancer immunoregulatory cytokines and a monoclonal antibody. It interacts with drugs and enhances the delivery of drugs. The carriers may be synthetic polymers thus deliver a high amount of administrated protein drug to cancer cells. It avoids accumulation in lower tissues and reduces toxicity. Polymeric micelles are copolymer has high loading 
capacity and easily modifiable surface features. These are best suited to deliver the chemical entities which have poor solubility. Their hydrophobic region interacts and the hydrophilic shell contributes toward solubility [16].

\section{Nanovaccines}

A vaccine has tremendous importance in oncology immunotherapy such as vaccine associated with adjuvant and cancer-associated antigens or cancer-specific neoantigens. Nanovaccines have the ability to deliver to secondary lymphoid organs i.e, lymph nodes [28]. They also show penetration to tissues hurdles, efficient intracellular delivery of nanoparticles, slow release of vaccine and antigens cross penetration in antigen presenting cells. Nanovaccine target to lymphoid modes recognize the receptors in immune cells and delivered molecular adjuvant so that triggers antigen-specific $\mathrm{T}$ cells response. They improve the efficiency of delivery by coordinating to lymphoid nodes residing lymphocytes this due to the pressure gradient between the blood and lymphatic system. Nanovaccines delivered the interstitial nanoparticles to LNs depend on the size, charge, hydrophilicity, and elasticity [29]. Nanovaccine delivered medium-size 5-100 nm nanoparticles to LNs. Nanovaccines can co-deliver multiepitope antigens. Synthetic nano vaccine includes organic and inorganic nanoparticles, eg: spherical nucleic acid with gold nanoparticle core and she'll of $\mathrm{CpG}$, a Toll-like receptor9 agonist developed for the destruction of the tumor. DNA nano vaccine develops by inorganic and organic scaffold for denaturation of a tumor. Naturally derived nano vaccines how good compatibility over synthetic nano vaccine naturally derived nanomaterial such as exogenous vaccine and endogenous vaccine prepared in vivo show efficient LNs targeted delivery. Such biomolecules show prolongs half-life. Nano vaccines deliver antigen and adjuvant to APCs efficiently and activate downstream signaling pathways. They show better biocompatibility than that of the free vaccine due to the multivalent adjuvant binding receptor, high cellular uptake efficiency and shielding of hydrophobicity. Thus induce a broad anticancer T-cell response with minimal immune tolerance but still show few challenges. Cancer immunotherapy tried to overcome the hurdle such as multi trimmer suppressive mechanisms with the collaboration of nano vaccine [18]. Trough nano vaccines antigen delivery is done efficiently. It ensures the sufficient concentration of antigen in antigen presenting cells APCs, sustain release of antigen and cytoplasmic delivery of antigen for MHC class 1 pressing. Nanotechnology also incorporates adjuvant and antigen in the same vehicles [17].

\section{Nanoshells}

Nanoshells are made by oppositely charged nanoparticles such as silica core surround by a metal shell having a diameter of $10-300 \mathrm{~nm}$ depend on the size of silica core. Gold nano-shells because of their favorable optical and chemical properties are used in biomedical research. They show the absorbance of infrared causing thermal destruction of cells [30].

\section{Carbon Nanotubes}

Carbon nanotubes having unique electrical, optical and mechanical properties that helps in diagnostic applications. Functionalization of carbon nanotube making them soluble in biological fluids and help to carry drug molecules, proteins, and nucleic acids at therapeutic interest [31]. Carbon nanotubes are made up of thin sheets of a benzene ring having mechanical, electrical and optical properties. They have the ability to convert near infrared and causes thermal destruction of cells. They are effectively used in breast cancer therapy [24]. CNT can be used as a nano-electronic biosensor to detect various biomolecules and also act as a vector to deliver the drug. Variety of factor affects the toxicity and biocompatibility of CNTs, such as a type of CNTs, impurities, length of CNTs [32]. The efficiency and toxicity of CNTs related to the characteristics and properties of the drug used to functionalize the CNTs. Functionalization can be done by using two approaches such as covalent attachment of functional group with other molecules of interest and noncovalent attachment that utilized hydrophobic interaction [20]. Many chemically modified CNTs have been used in many fields. A multivalent carbon nanotube drug delivery system functionalized and then conjugated through the natural compound 5,7, coumarin, to produce an MWNT-link-coumarin conjugate. That help to improve the bioactivity of 5,7coumarinby increasing its capability to reach the intracellular target. Different samples of carbon nanotubes can be obtained by carboxylation, acylation, amine modification and then combined with the natural compound for drug delivery [33].

\section{Quantum Dots}

Quantum dots are colloidal semiconductor nanocrystals consist of crystalline metalloids composed of zinc, cadmium, and selenium showing ability to target specific biomolecules. Quantum dots can deliver a higher amount of anti-tumor drugs [24]. Graphene quantum dots consider as carbon-based nanomaterial with increased potential in biomedical research or in cancer treatment [34]. With respect to graphene, QGD are less toxic and more hydrophobic and endowed of stable strong fluorescence [35]. Their intrinsic fluorescence is much 
more important for tracking the human cell in vitro. Graphene quantum dots (QGD) having a more attractive group that allow their multimodal conjugation on their surface making them ideal carrier for tracking of cancer. Due to unique properties of QGD over graphene or GO it helps to increase the chemotherapy efficiency of the anticancer drug [36]. QGD is synthesized by acidic oxidation and exfoliation of purified pristine multi-walled carbon nanotube [37]. QGD covalently bind to tumor targeting module biotin and recognize the biotin receptor that is overexpressed on cancer cells by means of a cleavable linker that are specifically activated in cells [38]. QGD surface is loaded with doxorubicin (DOX) that has excellent absorption properties by $p$-p interaction, moreover, that allowed the drug to be tracked [39].

\section{Nanohydrogels}

These are the 3D network of the cross-linked hydrophilic polymer have high content and size is less than 200nm. Formulation of hydrogels along with nanocarriers has delivered through physical interactions. They show high biocompatibility along prolongation survival [8]. They are lipo spherical nanocarriers having a diameter of $10-10,000 \mathrm{~nm}$. They are formulated by liposomes along with polymeric nanoparticles and show high stability. They are loaded with the hydrophobic and hydrophilic agent for better delivery [8].

\section{Biodegradable and Biomimic}

These are preferred over nonbiodegradable due to non-immunogenic having less toxicity. They mimic like biological component thus reduce side effect [16].They includes carbon nanotubes or graphene oxide, gold nanoparticles and magnetic nanoparticles. The size of the nanoparticles describe their characteristics features and used along with the therapeutic agent [8]. These are used in MRI. Super paramagnetic iron oxide nanoparticles are widely used. Some others like Gold and copper sulfide nanoparticles are used in Computed Tomography and Photochemical ablation therapy. With little modifications, they can be made site target specific to APCs [16]. Gold nanoparticles have the ability to scatter visible light. They are used in photo account stictomography. In vivo imaging of gold nanoparticle used in novel experimental therapies [4].

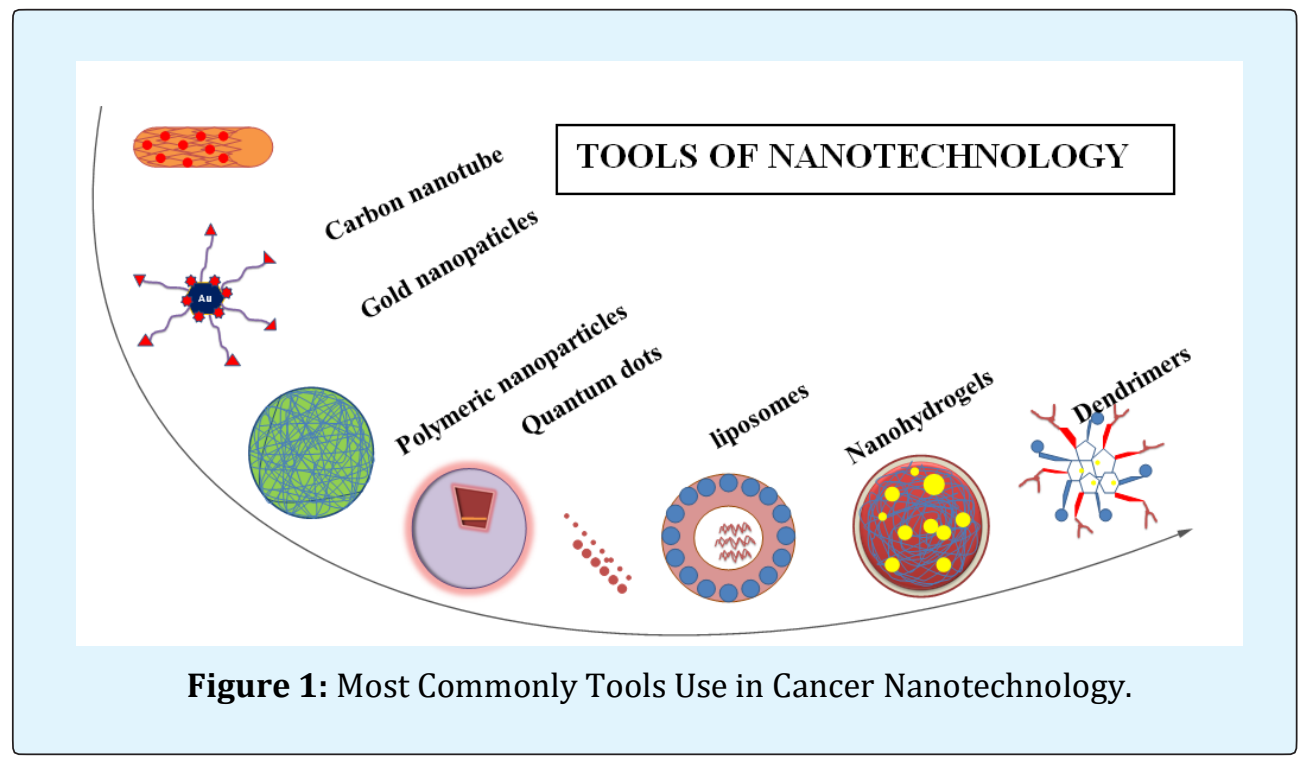

\section{Drug Delivery System}

\section{Passive Tumor Targeting}

In this type of targeting due to impaired lymphatic system and leaky surface of tumor vessel, PNPS easily reach infection tumor site and drug leads nanoparticles to accumulate in the tumor (interstitial space) this effect called as enhanced permeability and retention effect (EPR). In this type of delivery type of tumor delivery specify the drug delivery, eg: the vasculature preferentially allows the entry of the NPs and their EPR. According to tumor specificity, a drug and tumor-specific conjugate develops depend on the tumor embodiments [24]. Cancerous cells have rapid vascularization which causes leakage in architecture and impaired lymphatic drainage, thus allow more EPR. Nanoparticles targeting to tumor site should be specific size and properties for the maximum circulation. The surface should be hydrophilic so that avoids plasma protein adsorption and aggregation 
the idea behind this targeting in the vasculature of the tumor [4]. The defective angiogenesis of the vessels having no compact packaging of the endothelial cells provide entry of molecules but the method is still challenging. For example, all the tumor vessels don't have similar permeability which may affect the efficiency of drugs [40].

\section{Active Tumor Targeting}

In this type of delivery system, the nanoparticles directly target on to the surface of the receptor found in tumor cells. It can identify the receptor and bind. These receptors target by antibody conjugate nanoparticles, carbohydrate targeting or antigen directed targeting [24]. In this type of targeting the nanoparticles conjugation done with targeting components. Thus the nanoparticles accumulate in tumor cells. Specific interaction of nanoparticles like lectin-carbohydrates, antigen-antibody requires to achieve therapeutic results of this method overcomes the limitations of passive targeting because the drug is loaded along with cargo moiety [4]. This is a more successful approach than passive targeting. This is particularly used to eliminate CSCs which are loaded inside a tumor bed [40].

Inactive tumor targeting through receptor-mediated endocytosis the cell ternary structure enters the tumor cell ternary structure composed of a polymer, active chemotherapeutic drug and also ligands as a targeting moiety specific type of receptors are such as folate receptors (FRS) the epidermal growth factor receptor (EGFRS) and transferrin receptor (TFRS) mostly present on cancer cells are expressed on cancer or tumor cell that help ligands for their attachment, ligands are used to functionalize the drug led PNPS provides the required dose of anticancer agent to the tumor site for a sustained period of time [26].

\section{Challenges/Disadvantages}

Nanotechnology-based on imaging provides a noninvasive investigation of cancer. $58 \%$ of examine the first approach against breast cancer which is based on nanotechnology tools for imaging. Nanoparticles with high magnetization and nanosize medicine used in radiotherapy. The surface modifications are done to make them nontoxic and biocompatible [5]. But still, there are some challenges have to face the cancer nanotechnology. To prevent non-specific interactions with serum protein the nanocarrier having a special coating, that tags them for internalization by the cell within the reticuloendothelial system (RES). This increase the circulation time of nanocarrier, reduce immunogenicity and nonspecific uptake by cell with (RES) [6]. Cancer therapy challenges include systematic distribution of antitumor agent, inadequate drug concentration reaching the tumor site, intolerable cytotoxicity. Limited ability to monitor therapeutic responses and development of multiple drug resistance. So there is a need to develop the methods or techniques to treat cancer. So cancer nanotechnology provides help in this respect. For cancer therapy, we need a therapeutic agent that acts only on the cancer site and not damage the normal cell. In this regard, several techniques developed such ad ligand-targeted therapeutic strategies including immunotoxins, radioimmunotherapy and drug immunoconjugates [1].

\section{Conclusion}

Cancer the uncontrolled division of cells occurs due to genetic instability and alteration. Heterogeneity of tumor makes the cell more complex Cancer is still an incurable ailment in the pregenomic era the main focus of treatment is on alterations of biochemical pathways, not on the genetic instability that leads to $13 \%$ of death worldwide. Nanotechnology has become the powerful tool in treatment in the treatment of cancer. Though there are some typical issues related to nanotechnology are long term toxicity, degradation, and metabolism of nanotechnology agents. Researchers are trying to overcome these hurdles so that nanotechnology can show promising results in the treatment of cancer.

\section{References}

1. Misra R, Acharya S, Sahoo SK (2010) 'Cancer nanotechnology: application of nanotechnology in cancer therapy'. Drug Discovery Today 15(19-20): 842-850.

2. Currell F, Curley S, Krpetić Ž, Bellringer M (2017) 'From basics to clinic: Cancer Nanotechnology. Cancer Nanotechnol 8(1): 8.

3. Salk JJ, Fox EJ, Loeb LA (2010) Mutational heterogeneity in human cancers: origin and consequences. Annu Rev Pathol 5: 51-75.

4. Kolhe S, Parikh K (2012) Application of nanotechnology in cancer: a review. Int J Bioinform Res Appl 8(1-2): 112-125.

5. Avitabile E, Bedognetti D, Ciofani G, Bianco A , Delogu LG (2018) How can nanotechnology help the fight against breast cancer? Nanoscale 10(25): 1171911731. 
6. Zeineldin R, Syoufjy J (2018) Cancer Nanotechnology 'Chapter 1'. Springer 1530: 3-12.

7. Ryan SM, Mantovani G, Wang X, Haddleton DM, Brayden DJ (2016) 'Advances in PEGylation of important biotech molecules: delivery aspects. Expert Opin Drug Deliv 5(4): 371-383.

8. Zhang $X$, Zhang $P$ (2016) Nanotechnology for multimodality treatment of cancer. Oncol Lett 12(6): 4883-4886.

9. Javed M, Ahamed M, Alhadlaq HA, Alrokayan SA, Kumar S (2014) Targeted anticancer therapy: Overexpressed receptors and nanotechnology. Clinica Chimica Acta 436: 78-92.

10. Brannon-peppas L, Blanchette JO (2009) Corrigendum to Nanoparticle and targeted systems for cancer therapy. Advanced Drug Delivery Reviews 61(4): 364 .

11. Campani V, Salzano G, Lusa S, De Rosa G (2016) Lipid Nanovectors to Deliver RNA Oligonucleotides in Cancer. Nanomaterials (Basel) 6(7): 1-22.

12. Ferrari M (2005) Cancer Nanotechnology: opportunities and challenges. Nature Reviews Cancer 5: $161-171$.

13. Hartshorn CM, Bradbury MS, Lanza GM, Nel AE, Rao J, et al. (2018) Nanotechnology Strategies to Advance Outcomes in Clinical Cancer Care. ACS Nano 12(1): 24-43.

14. Mi Y, Shao Z, Vang J, Kaidar-Person O, Wang AZ (2016) Application of nanotechnology to cancer radiotherapy. Cancer Nanotechnol 7(1): 11.

15. Siddiqui IA, Sanna V (2016) Impact of nanotechnology on the delivery of natural products for cancer prevention and therapy. Mol Nutr Food Res 60(6): 1330-1341.

16. Cheng C, Castro G, Liu CH, Lau P (2019) Advanced nanotechnology: An arsenal to enhance immunotherapy in fighting cancer. Clin Chim Acta 492: 12-19.

17. Gharpure KM, Wu SY, Li C, Lopez-Berestein G, Sood AK (2015) 'Nanotechnology: Future of Oncotherapy'. Clin Cancer Res 21(14): 3121-3130.

18. Zhu G, Zhang F, Ni Q, Niu G, Chen X, et al. (2017) Efficient Nanovaccine Delivery in Cancer Immunotherapy. ACS Nano 11(3): 2387-2392.
19. King RB, McMahon SJ, Hyland WB, Jain S, Butterworth KT, et al. (2017) An overview of current practice in external beam radiation oncology with consideration to potential benefits and challenges for nanotechnology. Cancer Nanotechnology 8(1): 3.

20. Imran M, Jamshaid U, Jamshaid T, Zafar N, Fessi H, et al. (2016) Carbon nanotubes from synthesis to in vivo biomedical applications. Int J Pharm 501(1-2): 278299.

21. Chen XJ, Zhang XQ, Liu Q, Zhang J, Zhou G (2018) 'Nanotechnology : a promising method for oral cancer detection and diagnosis', J Nanobiotechnology 16(1): 52 .

22. Krishnamurthy S, Vaiyapuri R, Zhang L, Chan JM (2015) 'Biomaterials Science Lipid-coated polymeric nanoparticles for'. Biomater Sci 3(7): 923-936.

23. Malam Y, Loizidou M, Seifalian AM (2009) Liposomes and nanoparticles: nanosized vehicles for drug delivery in cancer. Trends Pharmacol Sci 30(11): 592599.

24. Singh SK, Singh S, Lillard JW, Singh R (2017) Drug delivery approaches for breast cancer. Int J Nanomedicine 12: 6205-6218.

25. Yang S, Gao H (2017) Nanoparticles for modulating tumor microenvironment to improve drug delivery and tumor therapy. Pharmacol Res 126:97-108.

26. Masood F (2016) Polymeric nanoparticles for targeted drug delivery system for cancer therapy. Materials Science \& Engineering C 60: 569-578.

27. Mendes LP, Pan J, Torchilin VP (2017) Dendrimers as Nanocarriers for Nucleic Acid and Drug Delivery in Cancer Therapy. Molecules 22(9): 1-21.

28. Luo M, Samandi LZ, Wang Z, Chen ZJ, Gao J (2018) Synthetic nanovaccines for immunotherapy. J Control Release 263: 200-210.

29. Song W, Musetti SN, Huang L (2017) Nanomaterials for Cancer Immunotherapy. Biomaterials 148: 16-30.

30. Wang YC, Rhéaume É, Lesage F, Kakkar A (2018) Synthetic Methodologies to Gold Nanoshells. Molecules 23(11): 1-28.

31. Amenta V, Aschberger K (2014) Carbon nanotubes: potential medical applications and safety concerns. 7(3): 371-386. 
32. Cui H, Vashist SK, Al-Rubeaan K, Luong JH, Sheu FS (2010) Interfacing Carbon Nanotubes with Living Mammalian Cells and Cytotoxicity Issues. Chem Res Toxicol 23(7): 1131-1147.

33. Iannazzo D, Piperno A, Ferlazzo A, Pistone A, Milone C, et al. (2012) Functionalization of multi-walled carbon nanotubes with coumarin derivatives and their biological evaluation. Org Biomol Chem 10(5): 1025-1031.

34. Chen F, Gao W, Qiu X, Zhang H, Liu L, et al. (2018) Graphene quantum dots in biomedical applications: Recent advances and future challenges. Frontiers in Laboratory Medicine 1(4): 192-199.

35. Lin L, Zhang S (2012) Creating high yield water soluble luminescent graphene quantum dots via exfoliating and disintegrating carbon nanotubes and graphite flakes. Chem Commun (Camb) 48(82): 10177-10179.

36. Sui X, Luo C, Wang C, Zhang F, Zhang J, et al. (2016) Graphene Quantum Dots Enhance Anticancer Activity of Cisplatin via Increasing Its Cellular and Nuclear Uptake. Nanomedicine 12(7): 1997-2006.

37. Lavin JG, Subramoneya S, Ruoff RS, Berber S, Tománek D (2002) Scrolls and nested tubes in multiwall carbon nanotubes. Carbon 40(7): 11231130.

38. Russell-jones G, McTavish K, McEwan J, Rice J, Nowotnik D (2004) Vitamin-mediated targeting as a potential mechanism to increase drug uptake by tumours. J Inorg Biochem 98(10): 1625-1633.

39. Iannazzo D, Pistone A, Salamo M, Galvagno S, Romeo $\mathrm{R}$, et al. (2017) Graphene quantum dots for cancer targeted drug delivery. Int J Pharm 518(1-2): 185192.

40. Ahmad G, Amiji MM (2017) Cancer stem cell-targeted therapeutics and delivery strategies. Expert Opinion on Drug Delivery 14(8): 997-1008. 